\title{
Influência da utilização de diferentes teores de lama vermelha nas propriedades de ladrilhos hidráulicos
}

\author{
The influence of using different red mud content in the \\ properties of hydraulic tiles
}

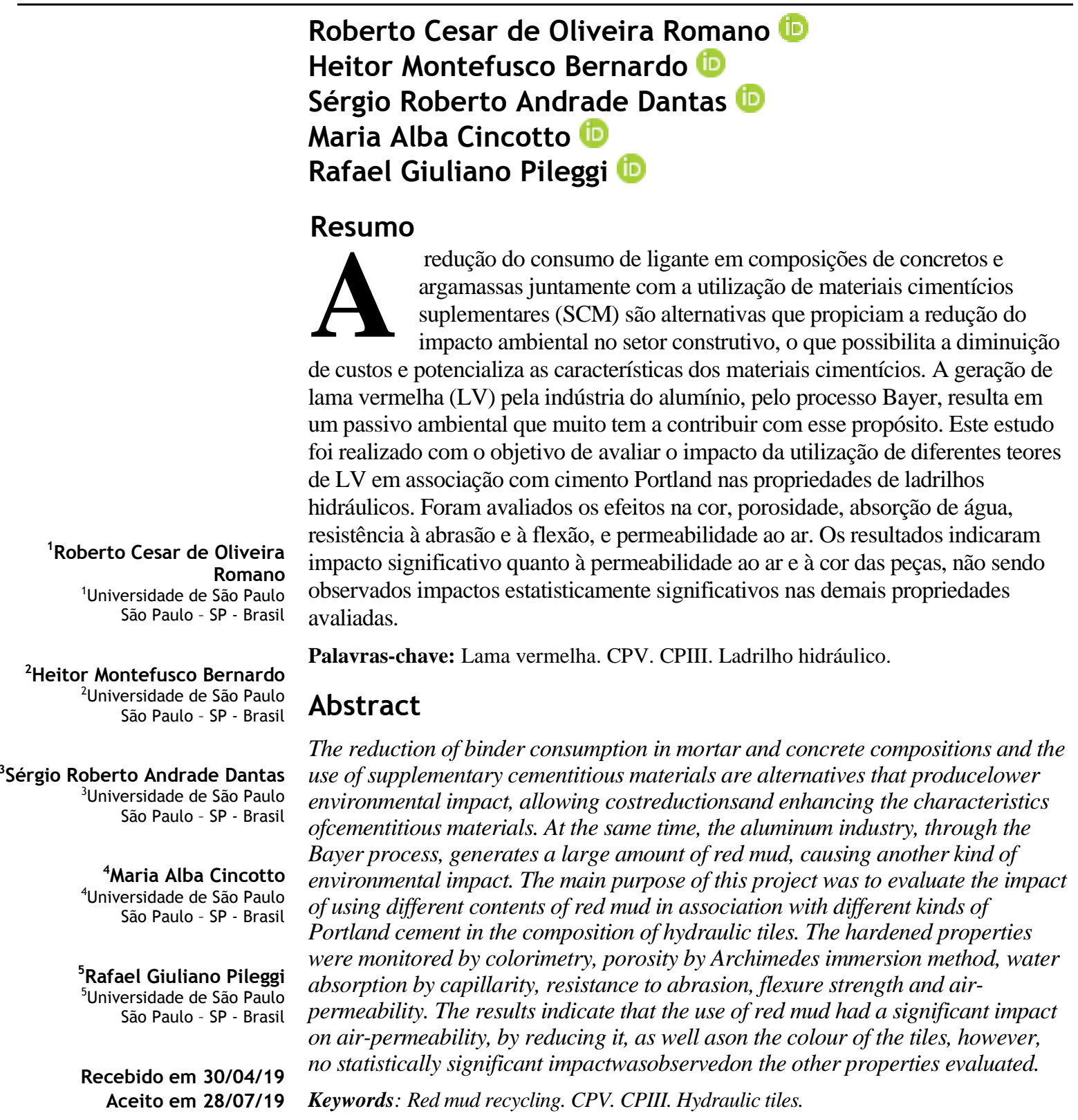

ROMANO, R. C. de O.; BERNARDO, H. M.; DANTAS, S. R. A.; CINCOTTO, M. A.; PILEGGI, R. G. Influência da utilizaçãode diferentes teores de lama vermelha nas propriedades de ladrilhos hidráulicos. 


\section{Introdução}

A produção de ladrilhos hidráulicos no Brasil data de meados do século XIX, mas, em função da falta de desenvolvimento da técnica de produção, os produtos passaram a perder espaço para os revestimentos cerâmicos (ASSOCIAÇÃO..., 2010; CAMPOS, 2011; CAVALLI; VALDUGA, 2006).

Atualmente, diante das condições de aplicação e uso, necessitam de melhor desempenho com relação à durabilidade e à resistência mecânica, geralmente alcançados com a utilização de grandes quantidades de cimento Portland (CATOIA, 2007; LOLLINI; REDAELLI; BERTOLINI, 2014).

Essa estratégia de dosagem, utilizada muitas vezes para a formulação de concretos e argamassas em geral, resulta em considerável impacto ambiental em função da elevada quantidade de $\mathrm{CO}_{2}$ liberado durante a produção do cimento (LOTHENBACH; SCRIVENER; HOOTON, 2011). Além disso, já está provado que a resistência dos materiais cimentícios nem sempre está relacionada com a quantidade de ligante utilizada nas formulações (ROMANO et al., 2017).

Visando reduzir as emissões de $\mathrm{CO}_{2}$, em diversos países são utilizadas adições minerais na fabricação do cimento ou substituição de parte do cimento por materiais suplementares (LOLLINI; REDAELLI; BERTOLINI, 2014; LOTHENBACH; SCRIVENER; HOOTON, 2011). As mais comuns, calcário, pozolanas e escória, tradicionalmente empregadas, possibilitam a diminuição do fator clínquer na produção ou consumo de cimento nas formulações sem perder desempenho e durabilidade em uso.

Por outro lado, existem materiais residuais que merecem estudo aprofundado, como a lama vermelha, gerada em elevada quantidade na cadeia de produção de alumínio metálico, que, acondicionada a céu aberto, causa considerável preocupação quanto ao problema ambiental que ela pode criar (LIBERATO et al., 2012; ROMANO et al., 2013, 2016).

Apresentando composição química e mineralógica com elevados teores de aluminatos e silicatos solúveis em água, a utilização em associação com cimento Portland pode ser uma alternativa de aplicação em larga escala para o rejeito desde que não sejam observados prejuízos nos estados fresco e endurecido (LIBERATO et al., 2012).

O objetivo deste trabalho foi avaliar a influência da utilização de diferentes teores de lama vermelha nas propriedades de ladrilhos hidráulicos, visando ao desenvolvimento de uma aplicação para o rejeito com redução do impacto ambiental tanto da produção do cimento quanto da geração do resíduo.

\section{Materiais e métodos de ensaio Caracterização das matérias-primas}

(a) granulometria a laser: a distribuição de tamanho de partículas foi determinada em um equipamento Sympatec, modelo Helos KR, com faixa de detecção de $0,1 \mu \mathrm{m}$ a $350 \mu \mathrm{m}$;

(b) granulometria por análise dinâmica de imagem: a distribuição de tamanho de partículas das areias foi determinada por análise dinâmica de imagens no equipamento QICPIC - Sympatec;

(c) picnometria de gás hélio: a densidade real dos pós foi determinada em um equipamento Quantachrome MVP 5DC Multipycnometer; e

(d) área superficial específica (ASE): essa análise foi realizada de acordo com o método de BET (Brunauer, Emmett e Teller), usando um equipamento Belsorp Max. As amostras foram tratadas previamente à temperatura de $60^{\circ} \mathrm{C}$ e pressão de vácuo de $10^{-2}$ psi por $24 \mathrm{~h}$ em um equipamento Belprep-vac II.

A distribuição granulométrica das partículas é apresentada na Figura 1, e a ASE e a densidade real dos pós, na Tabela 1.

Os materiais apresentam estreita faixa de variação da densidade. Contudo, a lama vermelha (LV) apresenta maior área superficial específica, devido à elevada quantidade de materiais zeolíticos, com diferentes morfologias, detectado a partir da determinação das fases mineralógicas por difração de raios X (resultados apresentados por Romano et al. (2016)).

Merece ser destacado que a LM foi recebida com cerca de $30 \%$ de umidade, e, por isso, foi necessária a secagem em estufa a $105^{\circ} \mathrm{C}$ por $24 \mathrm{~h}$. Após esse tempo, os torrões secos foram moídos em moinho de facas, e o produto peneirado em abertura de malha de $106 \mu \mathrm{m}$. Esse procedimento foi adotado para a adequação do material a ser aplicado como suplementar ao cimento.

648 Romano, R. C. de O.; Bernardo, H. M.; Dantas, S. R. A.; Cincotto, M. A.; Pileggi, R. G. 
Figura 1 - Distribuição granulométrica das matérias-primas utilizadas nas formulações dos ladrilhos hidráulicos

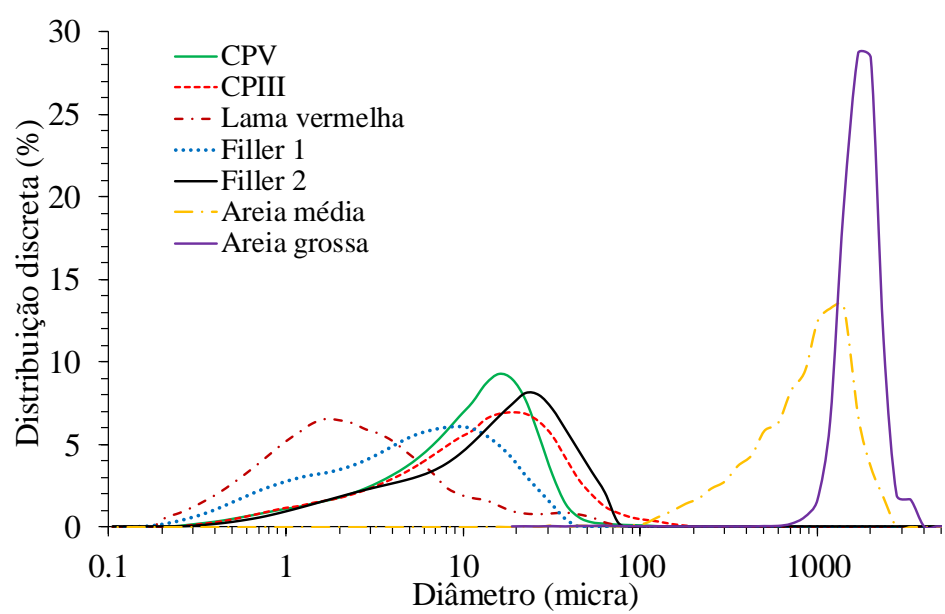

Tabela 1 - Área superficial específica (ASE) e densidade real das matérias-primas utilizadas nas formulações dos ladrilhos hidráulicos

\begin{tabular}{l|c|c}
\hline \multicolumn{1}{c|}{ Matéria-prima } & ASE $\left(\mathbf{m}^{2} / \mathbf{g}\right)$ & Densidade real $\left(\mathbf{g} / \mathbf{c m}^{3}\right)$ \\
\hline CPV & 1,20 & 2,99 \\
CPIII & 2,10 & 2,95 \\
Lama vermelha & 8,90 & 2,93 \\
Filler 1 & 2,02 & 2,86 \\
Filler 2 & 0,54 & 2,73 \\
Areia média & 0,30 & 2,60 \\
Areia grossa & 0,20 & 2,61 \\
\hline
\end{tabular}

As propriedades físicas da LV resultam em composições que, em teoria, devem demandar maior quantidade de água para a manutenção da trabalhabilidade durante a moldagem dos produtos. Neste estudo optou-se por manter a quantidade de água constante para avaliar puramente o impacto do resíduo no estado endurecido. Todas as composições apresentaram condições adequadas de aplicação, obtendo-se ladrilhos com excelente acabamento superficial.

\section{Composições avaliadas}

As composições utilizadas no estudo são apresentadas na Tabela 2. Os teores de LV foram dosados em massa, em relação ao teor de cimento. A quantidade de cimento nas formulações foi mantida constante utilizando-se fíler calcário (material inerte) para manter o volume de finos constante, e o aumento do consumo de LV foi realizado reduzindo-se a quantidade do material inerte. Essa estratégia foi adotada para manter o consumo de ligante constante em todas as composições. Um superplastificante à base de policarboxilato de sódio foi utilizado para reduzir a quantidade de água necessária para o amassamento.

\section{Moldagens e cura}

A mistura foi feita em uma argamassadeira planetária Hobart, modelo N50. Todo o pó foi depositado na cuba, e a água adicionada em seguida. Aguardaram-se $30 \mathrm{~s}$ para o início da molhagem dos pós. No primeiro estágio da mistura, o cisalhamento aplicado foi mantido na velocidade 1 do equipamento por 2 min. Após esse tempo, a agitação foi cessada para limpeza do recipiente e da hélice utilizada na mistura. No segundo estágio da mistura, a velocidade foi aumentada e mantida por mais $40 \mathrm{~s}$.

A suspensão obtida foi adicionada em moldes poliméricos com $20 \mathrm{~cm}$ de comprimento, $10 \mathrm{~cm}$ de largura e $1,5 \mathrm{~cm}$ de espessura. Foram moldados 6 ladrilhos para cada composição. Após a moldagem, todos os corpos de prova permaneceram 1 min sob vibração em mesa vibratória para remoção das bolhas remanescentes. Os produtos foram curados por 28 dias em câmara seca com controle da temperatura em $23 \pm 2{ }^{\circ} \mathrm{C}$ e umidade relativa de $50 \pm 3 \%$. 
Tabela 2 - Proporções (\%) das matérias-primas utilizadas nas composições dos pisos

\begin{tabular}{|c|c|c|c|c|c|c|c|c|}
\hline Matéria-prima & CPV & $\begin{array}{l}\text { CPV- } \\
5 \mathrm{LV}\end{array}$ & $\begin{array}{l}\text { CPV- } \\
10 L V\end{array}$ & $\begin{array}{l}\text { CPV- } \\
20 L V\end{array}$ & CPIII & $\begin{array}{c}\text { CPIII- } \\
\text { 5LV }\end{array}$ & $\begin{array}{l}\text { CPIII- } \\
\text { 10LV }\end{array}$ & $\begin{array}{l}\text { CPIII- } \\
20 \mathrm{LV}\end{array}$ \\
\hline $\mathrm{CPV}$ & \multirow{2}{*}{\multicolumn{4}{|c|}{20}} & \multicolumn{4}{|c|}{-} \\
\hline CPIII & & & & & \multicolumn{4}{|c|}{20} \\
\hline Lama vermelha & - & 1,0 & 2,0 & 4,0 & - & 1,0 & 2,0 & 4,0 \\
\hline Filler 1 & 12,0 & 11,0 & 10,0 & 8,0 & 12,0 & 11,0 & 10,0 & 8,0 \\
\hline Filler 2 & \multicolumn{4}{|c|}{8,0} & \multicolumn{4}{|c|}{8,0} \\
\hline Areia média & \multicolumn{4}{|c|}{33,0} & \multicolumn{4}{|c|}{33,0} \\
\hline Areia grossa & \multicolumn{4}{|c|}{27,0} & \multicolumn{4}{|c|}{27,0} \\
\hline Superplastificante* & \multicolumn{4}{|c|}{0,3} & \multicolumn{4}{|c|}{0,3} \\
\hline Água** & \multicolumn{4}{|c|}{13,0} & \multicolumn{4}{|c|}{13,0} \\
\hline
\end{tabular}

Nota: *porcentagem em função da massa de finos; e **porcentagem de água em função da massa total de pó.

\section{Propriedades avaliadas}

Colorimetria: a avaliação da cor foi realizada de acordo com a D2244-16 - Standard Practice for Calculationof Color Tolerances and Color Differences from Instrumentally Measured Color Coordinates (AMERICAN..., 2016), utilizando-se um espectrocolorímetro Color Guide Sphere d/8 ${ }^{\circ}$ spin, com medições de geometria de 45 circ./0, d/8, com área de medição de 400-700 nm, resolução espectral de $20 \mathrm{~nm}$, área fotométrica com resolução de $0-100 \%(0,01)$ e um observador padrão D65 com ângulo de abertura de $10^{\circ}$. O procedimento de leitura foi realizado conforme proposto por Dantas (2016).

Porosidade: determinada pelo método de imersão baseado no princípio de Arquimedes. Inicialmente, as massas das amostras secas (ms) foram anotadas, e os corpos de prova imersos em água e submetidos a vácuo por cerca de $2 \mathrm{~h}$, para forçar sua intrusão nos poros. Em seguida, as amostras foram pesadas imersas $\left(\mathrm{m}_{\mathrm{im}}\right) \mathrm{e}$ úmidas $\left(\mathrm{m}_{\mathrm{u}}\right)$, e as porosidades calculadas a partir das Equação 1 e Equação 2:

PA $(\%)=\frac{m_{u}-m_{i m}}{m_{u}-m_{s}} \times 100 \%$

Eq. 1

PT $(\%)=\left(1-\rho_{\text {REL }}\right) \times 100 \%$

Eq. 2

Onde:

PA é a porosidade aparente;

PT é a porosidade total; e

$\rho_{\text {REL }}$ é a densidade relativa do cimento (ou resultante dos pós que compõem as argamassas).

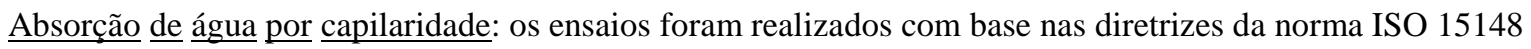
- Hygrothermal performance of building materials and products Determination of water absorption coefficient by partial immersion (INTERNATIONAL..., 2002). As bordas dos pisos foram seladas com cera de abelha, e as amostras colocadas em contato com uma película de água na superfície inferior, forçando a absorção do líquido somente por essa extremidade. Em cada tempo proposto, as amostras eram retiradas da bandeja, limpas para eliminação do excesso de água e pesadas. O coeficiente de permeabilidade (W), expresso em $\mathrm{kg} / \mathrm{m}^{2} \cdot \mathrm{h}^{1 / 2}$, foi calculado a partir da Equação 3 .

$W=\frac{m_{t}-m_{i}}{A \times \sqrt{t}}$

Onde:

A é a área $\left(\mathrm{m}^{2}\right)$;

t é o tempo em horas; e

$\mathrm{m}_{\mathrm{t}}$ e $\mathrm{m}_{\mathrm{i}}$ são as massas, em quilogramas, nos tempos de pesagem e inicial respectivamente.

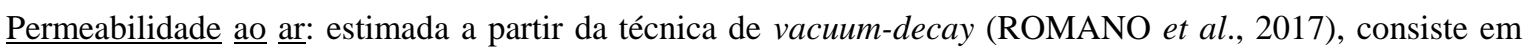
uma bomba de vácuo ligada a uma câmara de sucção de nylon que fica em contato com uma superfície da amostra, onde é formada, inicialmente, uma região de pressão negativa. Quando a bomba de vácuo é ligada, um transdutor registra as variações de pressão ao longo do tempo de ensaio, e os dados são coletados em uma placa aquisitora ligada ao computador. Com o desligamento da bomba de vácuo, o tempo de decaimento da pressão é quantificado, e os resultados de $\mathrm{k}_{1}$ obtidos a partir da equação de Forchheimer,

650 Romano, R. C. de O.; Bernardo, H. M.; Dantas, S. R. A.; Cincotto, M. A.; Pileggi, R. G. 
considerando, para efeito de simplificação, duas hipóteses: compressibilidade desprezível do gás e a utilização apenas do termo linear (INNOCENTINI et al., 2009).

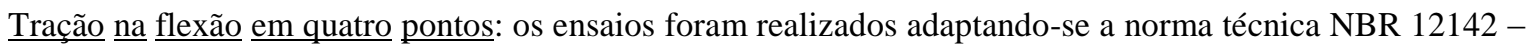
Concreto: Determinação da resistência a tração na flexão em corpos de prova prismáticos (ABNT, 2010). A distância entre os pontos de apoio foi de $130 \mathrm{~mm}$, e a distância entre os pontos de aplicação da carga, de 45 $\mathrm{mm}$. O teste foi realizado com controle de deslocamento de $1,5 \mathrm{~mm} / \mathrm{min}$.

Resistência à abrasão: os ensaios foram realizados com base na norma brasileira NBR 9781 - Peças de concreto para pavimentação - Especificação e métodos de ensaio (ABNT, 2013). O equipamento consiste em um disco rotativo de aço com diâmetro de $200 \mathrm{~mm}$ e espessura de $70 \mathrm{~mm}$, um suporte para o corpo de prova, um contrapeso, uma caixa de armazenagem de material abrasivo (alumina eletrofundida F80) e um funil de escoamento para a saída do material abrasivo. O corpo de prova é posicionado no equipamento, centralizado em relação ao centro do disco rotativo. Abastece-se o reservatório com o material abrasivo de modo que o fluxo seja constante com $100 \pm 0,05$ g a cada $100 \pm 5$ rotações do disco. Abre-se a válvula de controle para o material abrasivo e, simultaneamente, liga-se o motor configurado para atingir 75 revoluções em $60 \pm 3$ s. Retira-se o corpo de prova do equipamento e mede-se a espessura da cavidade. De acordo com os resultados obtidos, a amostra pode se enquadrar em duas faixas, conforme ilustrado na Tabela 3.

\section{Resultados e discussão}

\section{Avaliação da variação da cor}

Na Figura 2 são apresentados os pisos moldados com os diferentes cimentos e teores de lama vermelha. Foram avaliados quatro tipos de misturas com dois tipos de cimento quanto à variação de cor pelo método CIELab. As leituras foram realizadas em diferentes pontos visando identificar o impacto na alteração da cor das peças quando da adição da LV.

\section{Tabela 3 - Critérios para resistência à abrasão}

\begin{tabular}{l|c}
\hline \multicolumn{1}{c|}{ Solicitação } & Abrasão máxima (mm) \\
\hline Tráfego de pedestre, tráfego leve e tráfego médio & $\leq 23$ \\
\hline $\begin{array}{l}\text { Tráfego meio pesado, tráfego pesado, } \\
\text { tráfego muito pesado e solicitações especiais }\end{array}$ & $\leq 20$ \\
\hline
\end{tabular}

Figura 2 - Pisos moldados com os diferentes cimentos e teores de lama vermelha

CPIII
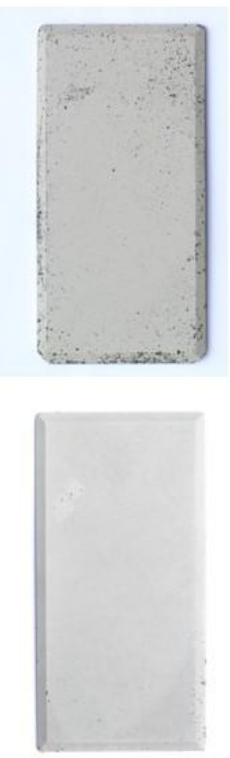

CPV
CPIII-5LV
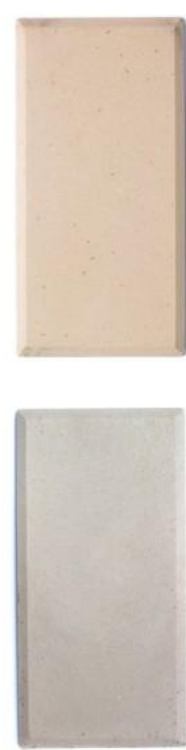

CPV-5LV
CPIII-10LV
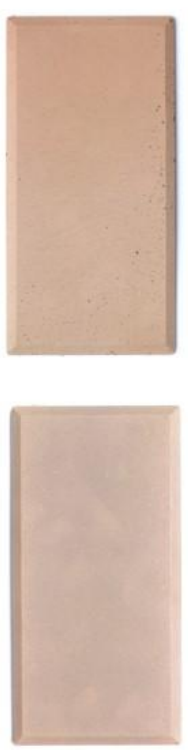

CPV-10LV
CPIII-20LV
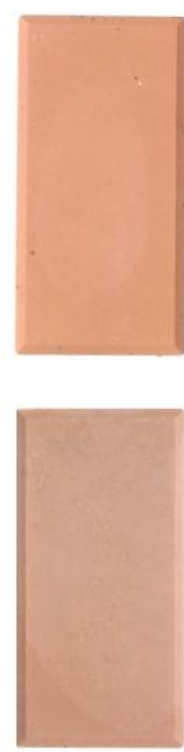

CPV-20LV 
A estabilidade da cor é uma característica importante para a comercialização de qualquer produto cuja falta de padronização possa causar prejuízo estético, levando à rejeição pelo consumidor final.

A descrição da cor é um processo de difícil definição devido aos muitos fatores que influenciam a percepção final por cada ser humano. A percepção humana da cor é complexa e abrange fenômenos subjetivos e objetivos. Portanto, a avaliação por comparações visuais pode não ser um método confiável, dados os resultados de inconsistências inerentes à percepção de cores e especificações entre observadores.

A colorimetria é a técnica que busca descrever e quantificar a percepção da cor pelo cérebro humano. A cor é expressa por três valores numéricos no CIE L* $a^{*} b^{*}$ : L, para a luminância, expressa em percentagem (de 0 para o preto a 100 para o branco); a e b, para as duas gamas de cor, que vão, respectivamente, do verde ao vermelho e do azul ao amarelo, com valores que vão de $-128 \mathrm{a}+128$. Assim, o modelo $\mathrm{L}^{*} \mathrm{a}^{*} \mathrm{~b}^{*}$ cobre a totalidade do espectro visível pelo olho humano e o representa de maneira uniforme.

A Figura 3 mostra as diferenças encontradas para os diferentes tipos de cimento e teores de LV avaliados. Os resultados obtidos por meio do espectrocolorímetro demonstram que, na comparação dos tipos de cimento usados nas misturas, o CPV foi o que demonstrou menor impacto na diferença de cor em relação à amostra de referência, independentemente do teor de LV utilizado.

A avaliação dos teores de LV demonstra que a menor diferença de cor das peças em relação à peça de referência é para o teor de 5\% de LV, independentemente do tipo de cimento utilizado.

Para os demais teores, os resultados indicam que, a partir dos $10 \%$ de adição da $\mathrm{LV}$, não há diferença significativa para o cimento CPV e há elevação da diferença de cor para o cimento do tipo CPIII.

Assim, diante de um parâmetro estipulado para o menor impacto na diferença de cor causado pela adição da LV nas peças, pode-se afirmar que a mistura CPV-5LV é a de melhor desempenho. Contudo, a falta de uma delimitação clara entre os critérios de avaliação visuais e a diferença de cor por colorimetria instrumental indica que outros fatores, além da diferença de cor por si só, podem influenciar na percepção visual (SHARMA; WU; DALAL, 2004; SOUZA, 2009).

Figura 3 - Diferença entre os tipos de cimento e os teores de lama vermelha avaliados
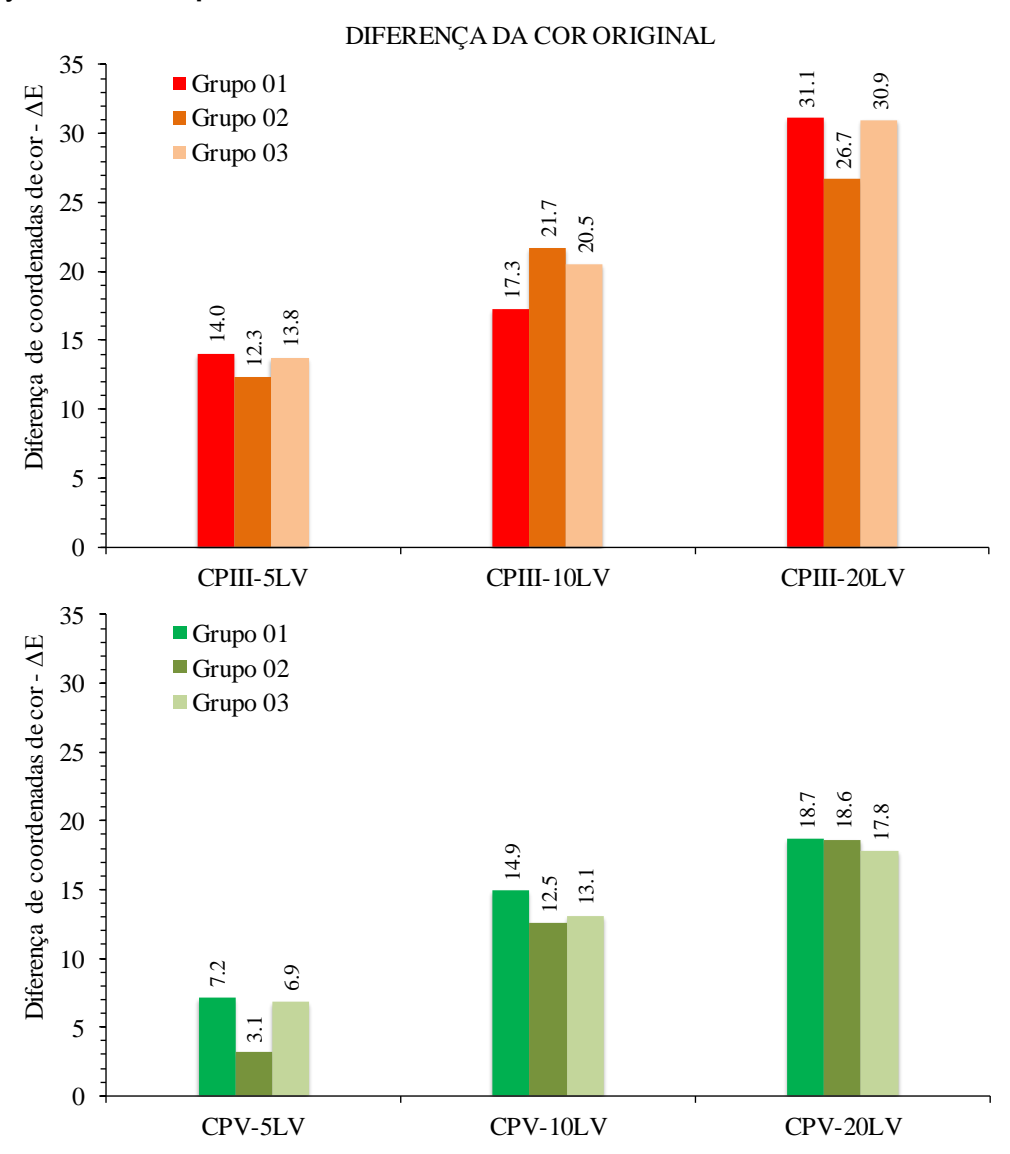

652 Romano, R. C. de O.; Bernardo, H. M.; Dantas, S. R. A.; Cincotto, M. A.; Pileggi, R. G. 
$\mathrm{O}$ delta $\mathrm{E}-\Delta \mathrm{E}$ ou dE (diferença total de cores) - é baseado em diferenças de cor $\mathrm{L}^{*} \mathrm{a}^{*} \mathrm{~b}^{*}$ e foi planejado para ser uma métrica de número único para decisões do tipo aceito ou rejeitado. Em uma escala típica, o valor do $\Delta \mathrm{E}$ varia de 0 a 100 , sendo:
$<1,0 \rightarrow$ não perceptivel pelos olhos humanos;
$1-2 \rightarrow$ perceptivel através de observação atenta;
2 a $10 \rightarrow$ perceptível num piscar de olhos;
11 a $49 \rightarrow \quad$ as cores são mais semelhantes do que opostas;
50 a $99 \rightarrow \quad$ as cores são mais opostas do que semelhantes; $e$
$100 \quad \rightarrow \quad$ as cores são exatamente o oposto.

Embora os resultados indicados pelo $\Delta \mathrm{E}$ possam ser utilizados como diferenciação de cores, devem ser tomados apenas como um guia geral, uma vez que é possível obter um valor $\Delta \mathrm{E}$ abaixo de 1,0 para duas cores que parecem diferentes. Esse é o caso das fórmulas CIE76 e CIE94, nas quais a saturação não é considerada ou não é ponderada adequadamente (BRAINARD, 2003; SHARMA; WU; DALAL, 2004).

Assim, devido às inconsistências entre os três algoritmos, o significado exato do $\Delta \mathrm{E}$ muda ligeiramente dependendo de qual fórmula é usada, o que o torna uma medida nem sempre confiável por si só, necessitando sempre da presença de uma análise visual como resposta final e, sobretudo, da delimitação quanto aos limites mínimos e máximos aceitáveis para uma aplicação específica.

A distância medida entre cores no espaço CIELab é uma boa aproximação da distância visual percebida Dessa forma, o limiar de percepção da cor conhecido como JND (Just Noticeable Difference) pode ser utilizado de forma a aumentar a correlação da avaliação objetiva com a avaliação subjetiva . No cálculo da distância entre cores, o JND é o limiar a partir do qual duas cores passam a ser percebidas como cores distintas pelo olho humano (DANTAS; VITTORINO; LOH, 2017).

De acordo com Souza (2009), não há um consenso sobre o valor preciso de JND , podendo assumir referência às faixas de valores conforme o cenário utilizado . Entre esses cenários assume -se um genérico e outro voltado para aplicaçõ es industriais. As faixas de valores JND para ambos são definidas conforme apresentado a seguir.

Em um cenário dito genérico tem-se que a percepção da unidade CIELab é diferenciada por:

$\begin{array}{lll}\text { entre } 0,38 \text { a } 0,73 & \rightarrow & \text { mínima diferença perceptivel ou JND; } \\ \text { em torno de } 1,75 & \rightarrow & \text { diferença supra JND; } \text { e } \\ \text { acima de } 5,0 & \rightarrow & \text { grandes diferenças de cor. }\end{array}$

Porém, a partir de uma perspectiva industrial, esses valores podem se alterar:

$\begin{array}{lll}\text { entre } 1,1 \text { e 2,8 } & \rightarrow & \text { tolerância de cor rigorosa; } \\ \text { entre } 2,9 \text { e 5,6 } & \rightarrow & \text { tolerância normal; } e \\ \text { acima de } 5,6 & \rightarrow & \text { tolerância folgada. }\end{array}$

Logo, tomando-se as faixas de JND apresentadas como referência e aplicando-as a uma produção em larga escala, ou seja, para a produção da cor em produtos manufaturados, tem-se que a composição CPV-5LV é a mistura que melhor atende ao objetivo pretendido, pois está dentro de um padrão de tolerância considerado normal para uma mudança de cor em escala industrial.

Os resultados demonstraram a importância da dosagem dos materiais para a produção das peças cimentícias, tendo em vista que os componentes cimentícios nem sempre são produzidos com o mesmo tipo de ligante ou, ainda, podem ser fabricados sem o preciso controle da dosagem das matérias-primas, resultando em consideráveis alterações na cor do produto.

\section{Porosidade e transporte de massa}

Outra característica de grande importância para a avaliação das características de ladrilhos hidráulicos é a porosidade do produto, pois em materiais cerâmicos, no geral, a resistência está relacionada com o volume de vazios estruturais.

Na Figura 4 são apresentados os resultados obtidos em função do tipo de cimento e teor de LV pelo ensaio de imersão de Arquimedes.

Para a avaliação da significância estatística da variação do teor de LV, foram utilizadas duas formas de análise: Anova com fator único, para rejeitar ou aceitar a hipótese da igualdade das médias, dentro e entre os 
grupos; e o teste Tukey, para avaliação comparativa entre pares com distintos teores de lama vermelha, de modo a indicar qual ou quais estão diferindo dos demais.

Os resultados obtidos a partir de Anova para a porosidade total são apresentados na Tabela 4. Acima dela estão os resultados para as pastas com o cimento CPIII, e abaixo, CPV.

Na primeira parte da tabela são apresentadas as informações da quantidade de amostras avaliadas para cada teor de LV, a soma dos resultados da porosidade total, a média dos resultados e a variância para cada caso. No entanto, somente com esses resultados não é possível avaliar se houve diferença estatística entre amostras em relação às porosidades (MACIEL et al., 2018).

Figura 4 - Porosidade total dos pisos, obtida a partir de ensaio de imersão de Arquimedes

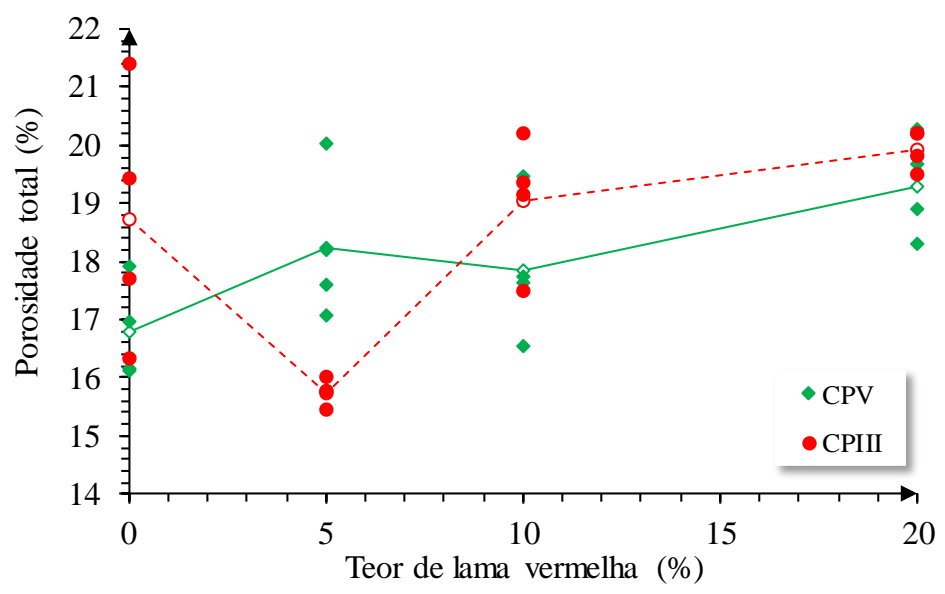

Tabela 4 - Anova fator único para avaliação estatística da variação da porosidade total CPIII

\begin{tabular}{|c|c|c|c|c|c|c|}
\hline Grupo & Contagem & Soma & Média & Variância & & \\
\hline Ref & 4 & 74,86 & 18,71 & 4,84 & & \\
\hline $5 \mathrm{LV}$ & 4 & 62,97 & 15,74 & 0,05 & & \\
\hline $10 \mathrm{LV}$ & 4 & 76,22 & 19,06 & 1,27 & & \\
\hline $20 \mathrm{LV}$ & 4 & 79,71 & 19,93 & 0,12 & & \\
\hline $\begin{array}{l}\text { Fonte da } \\
\text { variação }\end{array}$ & $S Q$ & $g l$ & $M Q$ & $F$ & valor-P & $F$ crítico \\
\hline Entre grupos & 39,70 & 3 & 13,23 & 8,43 & 0,0028 & 3.49 \\
\hline $\begin{array}{l}\text { Dentro dos } \\
\text { grupos }\end{array}$ & 18,83 & 12 & 1,57 & & & \\
\hline Total & 58,53 & 15 & & & & \\
\hline
\end{tabular}

\begin{tabular}{lcccc}
\multicolumn{1}{c}{ CPV } & & & & \\
\hline Grupo & Contagem & Soma & Média & Variância \\
\hline Ref & 4 & 67,16 & 16,79 & 0,73 \\
5LV & 4 & 72,91 & 18,23 & 1,68 \\
10LV & 4 & 71,37 & 17,84 & 1,43 \\
20LV & 4 & 77,15 & 19,29 & 0,75 \\
\hline
\end{tabular}

\begin{tabular}{lcccccc}
\hline \multicolumn{1}{c}{$\begin{array}{c}\text { Fonte da } \\
\text { variação }\end{array}$} & $S Q$ & $g l$ & $M Q$ & $F$ & valor-P & F crítico \\
\hline $\begin{array}{l}\text { Entre grupos } \\
\begin{array}{l}\text { Dentro dos } \\
\text { grupos }\end{array}\end{array}$ & 12,77 & 3 & 4,26 & $\mathbf{3 , 7 1}$ & 0,0425 & $\mathbf{3 . 4 9}$ \\
Total & 13,76 & 12 & 1,15 & & & \\
\hline
\end{tabular}

654 Romano, R. C. de O.; Bernardo, H. M.; Dantas, S. R. A.; Cincotto, M. A.; Pileggi, R. G. 
Já a segunda parte da tabela trata da fonte de variação entre os grupos. O valor-P indica o valor de prova e demonstra se a hipótese de igualdade entre os resultados referentes à variação do teor de resíduo nas composições deve ser aceita ou rejeitada. Se o valor de prova for superior ao erro, ou seja 0,05 , deve-se aceitar a igualdade; caso contrário, deve ser rejeitada. Nos casos avaliados, o valor foi de 0,0028 (CPIII) e de 0,0425 (CPV), indicando que em ambos os casos há diferença estatística entre as médias.

Outra forma de se concluir se há igualdade é comparar o valor de F com F crítico: o F crítico limita a região de rejeição e significa que, para valores de F superiores, a hipótese da igualdade deve ser rejeitada. Portanto, como o valor de $\mathrm{F}$ foi maior do que o de F crítico em ambos os casos, há indicativo de que o aumento do teor de resíduo resultou em alteração da porosidade total, e o valor-P menor do que 0,05 comprova a significância do resultado comparativo de F com F crítico.

No entanto, somente com o resultado da avaliação por Anova fator único não é possível saber qual ou quais composições são distintas a partir da avaliação em pares. Para tanto, utilizou-se a avaliação a partir do teste Tukey para comparação das variáveis duas a duas, e os resultados são apresentados na Tabela 5.

Dessa forma, ficou comprovado que a porosidade das amostras formuladas com o CPV somente foi distinta quando se utilizou $20 \%$ de substituição pelo resíduo de bauxita, e, para o caso das pastas com CPIII, as composições Ref, CPIII-10LV e CPIII-20LV são similares entre si e distintas da composição com substituição de cimento por $5 \%$ de lama vermelha.

Merece ser destacado que, como essa forma de avaliação foi realizada para as demais propriedades, as tabelas não serão mais apresentadas no texto; os resultados da avaliação estatística das demais propriedades serão discutidos diretamente.

A porosidade dos materiais cimentícios é uma propriedade de grande interesse prático, pois o volume de vazios na microestrutura, em teoria, apresenta relação inversamente proporcional às propriedades mecânicas, elásticas, superficiais e de transporte de massa dos produtos.

Na Figura 5 é apresentado o resultado do ensaio de absorção de água por sucção capilar. Os dados foram tratados conforme descrito por Carbone et al. (2011) e apresentados na forma de coeficiente de absorção de água $-\mathrm{W}$.

Fica claro que a presença da LV foi responsável pela melhora na estanqueidade dos pisos: quanto maior o teor utilizado nas composições, menor o valor de W. Essa é uma importante informação, pois se espera que o piso apresente baixa capacidade de absorção de água, como estratégia para aumentar a durabilidade, pois dificulta a penetração dos agentes de degradação, e para reduzir a tendência de liberação de íons por lixiviação.

Além da diminuição do valor de $\mathrm{W}$, o tempo necessário para a saturação dos pisos foi menor em função do aumento do teor de LV nas composições, e a partir da avaliação estatística foi comprovado que não há diferença ocasionada pela alteração do tipo de cimento, ou seja, para os mesmos teores de substituição, o valor de $\mathrm{F}$ foi sempre menor que $\mathrm{F}$ crítico no ensaio de Anova fator único. Por outro lado, o teor de resíduo apresentou variação estatisticamente considerável, e todos os teores são distintos entre si.

Tabela 5 - Teste Tukey para comparação das variáveis duas a duas - acima estão os resultados para as pastas com cimento CPIII, e abaixo, CPV - as células em destaque indicam diferença estatística

\begin{tabular}{lcccc}
\hline CPIII & Ref & $5 \mathrm{LV}$ & $10 \mathrm{LV}$ & $20 \mathrm{LV}$ \\
\hline Ref & & 0,0256 & 0,9796 & 0,5395 \\
$5 \mathrm{LV}$ & 4,746 & & 0,0131 & 0,0025 \\
$10 \mathrm{LV}$ & 0,5457 & 5,292 & & 0,7608 \\
20LV & 1,938 & 6,684 & 1,392 & \\
\hline \multicolumn{5}{c}{} \\
\hline CPV & Ref & $5 \mathrm{LV}$ & $10 \mathrm{LV}$ & $20 \mathrm{LV}$ \\
\hline Ref & & 0,2800 & 0,5290 & 0,0283 \\
$5 \mathrm{LV}$ & 2,682 & & 0,9558 & 0,5217 \\
$10 \mathrm{LV}$ & 1,964 & 0,7177 & & 0,2749 \\
$20 \mathrm{LV}$ & 4,664 & 1,9820 & 2,7000 & \\
\hline
\end{tabular}


Figura 5 - Coeficientes de absorção de água por capilaridade dos pisos

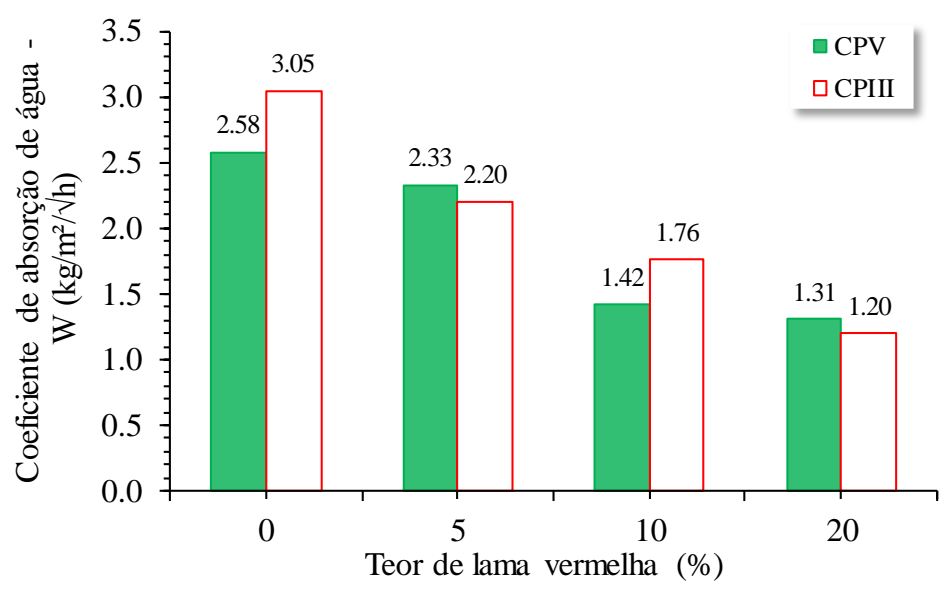

Outra forma de se avaliar o transporte de massa pela microestrutura do piso cimentício é a partir da quantificação da permeabilidade ao ar, e os resultados obtidos são ilustrados na Figura 6. Em todos os casos as médias são resultantes de leituras da permeabilidade em três amostras de cada teor de LV, e ficou muito claro que, para teores de lama acima de $10 \%$, houve diminuição da permeabilidade.

A permeabilidade ao ar dos materiais cimentícios apresenta íntima relação com a durabilidade dos produtos aplicados, visto que, quanto mais fácil a percolação de ar ou água, maior a facilidade para a penetração dos agentes de degradação.

Em ambos os casos, permeabilidade ao ar e absorção de água por capilaridade, foi observado que os resultados foram melhores para substituição acima de $10 \%$ de cimento por LV. Em função da distribuição granulométrica com maior quantidade de finos que do cimento, a substituição do ligante pela LV melhorou o empacotamento de partículas e diminuiu a quantidade de poros percoláveis, fato que pode aumentar a vida útil do produto.

\section{Avaliação mecânica}

A resistência mecânica dos materiais cimentícios pode apresentar relação com a quantidade de água adicionada durante a mistura das composições, pois a quantidade do líquido que não é utilizada para a reação do cimento é evaporada, o que gera poros microestruturais. No caso dos pisos avaliados neste projeto, o consumo de água foi mantido constante, sendo possível avaliar puramente o efeito da utilização da LV nas formulações, visto que esta foi a única variável no ensaio. Os resultados da resistência à flexão em quatro pontos são apresentados na Figura 7.

Não foi observada nenhuma tendência com relação ao teor de LV utilizado nem do tipo de cimento, visto que os resultados apresentaram elevada variabilidade. Essa é uma informação relevante porque foi comprovado que a utilização do resíduo não deteriorou a propriedade mecânica avaliada. A mesma observação é válida para o caso da resistência à abrasão, conforme apresentado na Figura 8. Todos os produtos avaliados poderiam ser utilizados em regiões de tráfego leve, conforme especificação da norma brasileira NBR 9781 - Peças de concreto para pavimentação - Especificação e métodos de ensaio (2013). Somente o piso de referência formulado com o cimento CPIII apresentou resistência fora dessa faixa de aplicação, ilustrando que a presença da LV melhorou a qualidade do produto.

\section{Conclusões}

Com base nos resultados obtidos, pode-se concluir que a utilização da LV não deteriorou as propriedades dos ladrilhos hidráulicos.

As alterações estatisticamente significativas foram observadas somente em relação à cor, permeabilidade ao ar e absorção de água por capilaridade, nesses dois últimos casos melhorando as propriedades dos pisos avaliados. Ambas as propriedades apresentam íntima relação com a durabilidade dos produtos cimentícios, pois afetam a capacidade de percolação dos agentes de degradação no interior das peças.

656 Romano, R. C. de O.; Bernardo, H. M.; Dantas, S. R. A.; Cincotto, M. A.; Pileggi, R. G. 
Figura 6 - Permeabilidade ao ar dos pisos com os distintos teores de LV

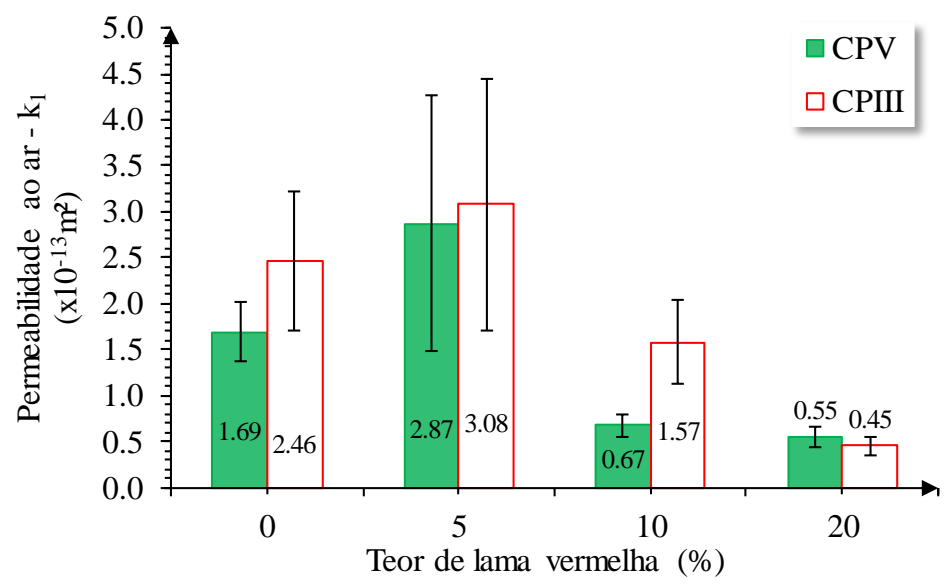

Figura 7 - Resistência a tração na flexão em quatro pontos dos pisos em função do teor de LV nas composições

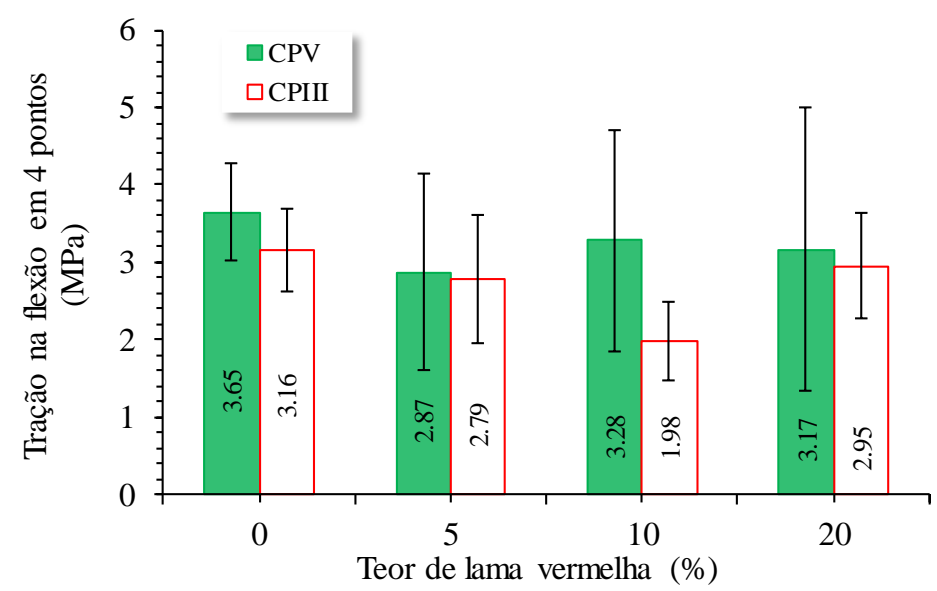

Figura 8 - Resistência à abrasão dos pisos com os distintos teores de lama vermelha

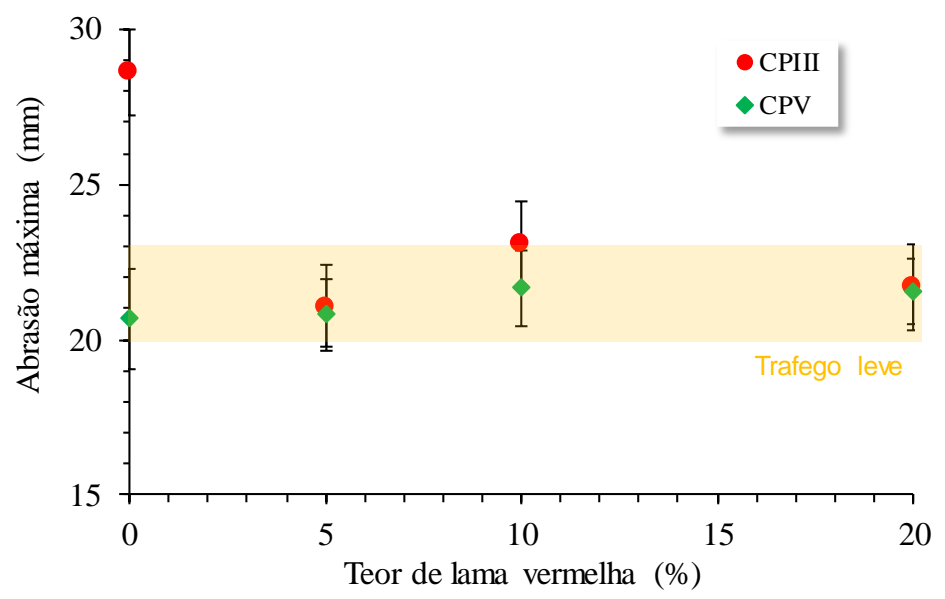

No caso da cor, foram observadas consideráveis alterações em função do teor de lama vermelha utilizada na composição e do tipo de cimento, sendo a composição CPV-5LV a que apresentou menor alteração de cor quando comparada à amostra de referência, praticamente imperceptível a olho nu. 
A avaliação mecânica apontou que o aumento do teor de substituição até $20 \%$ resultou em resistência à tração na compressão diametral e resistência à abrasão estatisticamente similares à amostra composta somente com cimento Portland.

Pode-se concluir que a utilização da lama vermelha em composições cimentícias depende tanto do teor do resíduo quanto do tipo de ligante utilizado na composição, pois as interações físico-químicas entre ambos seguem diferentes tendências, influenciando o desenvolvimento das propriedades no estado endurecido.

Por fim, deve ser ressaltado que, embora a lama vermelha apresente grande potencial de uso como um material cimentício suplementar, seu comportamento perante o processo de lixiviação precisa ser mais bem avaliado. Por isso, ensaios complementares estão sendo realizados para garantir que a prática possa ser realizada com sucesso.

\section{Referências}

AMERICAN SOCIETY FOR TESTING ANS MATERIALS. D2244-16: standard practice for calculation of color tolerances and color differences from instrumentally measured color coordinates. Pennsylvania, 2016.

ASSOCIAÇÃO BRASILEIRA DE CIMENTO PORTLAND. Manual ladrilho hidráulico. Disponível em: http://solucoesparacidades.com.br. Acesso em: 31 jul. 2017.

ASSOCIAÇÃO BRASILEIRA DE NORMAS TÉCNICAS. NBR 12142: concreto determinação da resistência a tração na flexão em corpos de prova prismáticos. Rio de Janeiro, 2010.

ASSOCIAÇÃO BRASILEIRA DE NORMAS TÉCNICAS. NBR 9781: peças de concreto para pavimentação: especificação e métodos de ensaio. Rio de Janeiro, 2013.

BRAINARD, D. H. 5 - Color Appearance and Color Difference Specification. The Science of Color (SecondEdition), p. 11-216, 2003.

CAMPOS, C. F. Trajetória e significado do ladrilho hidráulico. Belo Horizonte: Universidade Federal de Minas Gerais, 2011.

CARBONE, C. E. et al. Efeito da adição de látex nas pastas de cimento branco no estado endurecido. Ambiente Construído, Porto Alegre, v. 13, n. 3, p. 317-330, jul./set. 2011.

CATOIA, T. Ladrilhos e revestimentos hidráulicos de alto desempenho. São Carlos: Universidade de São Paulo, 2007.

CAVALLI, A. F.; VALDUGA, L. Ladrilhos hidráulicos: reconstituição e caracterização. In: ENCONTRO NACIONAL DE TECNOLOGIA NO AMBIENTE CONSTRUÍDO, 11., Florianópolis, 2006. Anais [...] Florianópolis, 2006.

DANTAS, S. R. A. A eficácia da adição do $\mathbf{T i O}_{2}$ em argamassas quanto à manutenção das condições iniciais em termos da sua refletância à radiação solar. São Paulo, 2016. Dissertação (Mestrado Profissional em Habitação: Planejamento e Tecnologia) - Instituto de Pesquisas Tecnológicas, São Paulo, 2016.

DANTAS, S. R. A.; VITTORINO, F.; LOH, K. The effectiveness of $\mathrm{TiO}_{2}$ additions to mortar to maintain initial conditions in terms of its reflectance to solar radiation. Ambiente Construído, Porto Alegre, v. 17, n. 3, p. 39-56, jul./set. 2017.

INNOCENTINI, M. D. M. et al. Permeability optimization and performance evaluation of hot aerosol filters made using foam incorporated alumina suspension. Journal of Hazardous Materials, v. 162, p. 212-221, 2009.

INTERNATIONAL ORGANIZATION FOR STANDARDIZATION. ISO 15148: hygrothermal performance of building materials and products: determination of water absorption coefficient by partial immersion. Switzerland, 2002.

LIBERATO, C. C. et al. Effect of bauxite residue calcination on rheological and hardened properties of suspensions with Portland cement. Ambiente Construído, Porto Alegre, v. 12, n. 3, p. 7-23, jul./set. 2012.

LOLLINI, F.; REDAELLI, E.; BERTOLINI, L. Effects of Portland cement replacement with limestone on the properties of hardened concrete. Cement and Concrete Composites, v. 46, p. 32-40, 2014. 
LOTHENBACH, B.; SCRIVENER, K.; HOOTON, R. D. Supplementary cementitious materials. Cement and Concrete Research, v. 41, n. 12, p. 1244-1256, 2011.

MACIEL, M. H. et al. Efeito da variação do consumo de cimento em argamassas de revestimento produzidas com base nos conceitos de mobilidade e empacotamento de partículas. Ambiente Construído, Porto Alegre, v. 18, n. 1, p. 245-259, jan./mar.2018.

ROMANO, R. C. O. et al. Acompanhamento da hidratação de cimento Portland simples com resíduo de bauxita. Revista Cerâmica, v. 62, p. 215-223, 2016.

ROMANO, R. C. O. et al. Evaluation of transition from fluid to elastic solid of cementitious pastes with bauxite residue using oscillation rheometry and isothermal calorimetry. Applied Rheology, v. 23, p. 1-15, 2013.

ROMANO, R. C. O. et al. Impact of using bauxite residue in microconcrete and comparison with other kind of supplementary cementitious material. In: INTERNATIONAL ICSOBA CONFERENCE, 35., Hamburg, 2017. Proceedings [...] Hamburg, 2017.

SHARMA, G.; WY. W.; DALAL, E. N. The CIEDE2000 Color-Difference formula: implementation notes, supplementary test data, and mathematical observations. Color Research and Application, v, 30, p. 21-30, 2004.

SOUZA, R. C. C. Avaliação de imagens através de similaridade estrutural e do conceito de mínima diferença de cor perceptível. Rio de Janeiro, 2009. Dissertação (Mestrado em Engenharia Eletrônica) Escola de Engenharia, Universidade do Estado do Rio de Janeiro, Rio de Janeiro, 2009.

\section{Agradecimentos}

Os autores agradecem ao Laboratório de Microestrutura e Ecoeficiência de Materiais e à Alcoa Foundation pelo apoio financeiro, à CAPES pela bolsa de pós-doutorado, e à Fapesp (Projeto 2014/50948-3 - INCT CEMtec - Tecnologias CimentíciasEcoeficientes Avançadas) pelo apoio durante a realização do trabalho.

Roberto Cesar de Oliveira Romano

Departamento de Construção Civil, Escola Politécnica | Universidade de São Paulo | Av. Professor Almeida Prado, Trav. 2, 83 | São Paulo - SP- Brasil | CEP 05508-010 | Tel.: (11) 3091-5248 | E-mail: rcorjau@gmail.com

\section{Heitor Montefusco Bernardo}

Departamento de Construção Civil, Escola Politécnica | Universidade de São Paulo | Tel.: (11) 3091-5283 | E-mail: heitor.bernardo@Ime.pcc.usp.br

\section{Sérgio Roberto Andrade Dantas}

Departamento de Construção Civil, Escola Politécnica | Universidade de São Paulo | Tel.: (11) 99884-3664 | E-mail: sergiodantas@usp.br

Maria Alba Cincotto

Departamento de Construção Civil, Escola Politécnica | Universidade de São Paulo | Tel.: (11) 3091-5283 | E-mail: cincotto@lme.pcc.usp.br

\section{Rafael Giuliano Pileggi}

Departamentode Construção Civil, Escola Politécnica | Universidade de São Paulo | Tel.: (11) 3091-5442 | E-mail: rafael.pileggi@lme.pcc.usp.br

\section{Ambiente Construído}

Revista da Associação Nacional de Tecnologia do Ambiente Construído Av. Osvaldo Aranha, 99 - $3^{\circ}$ andar, Centro

Porto Alegre - RS - Brasil CEP 90035-190

Telefone: +55 (51) 3308-4084

Fax: +55 (51) 3308-4054

www.seer.ufrgs.br/ambienteconstruido

E-mail: ambienteconstruido@ufrgs.br

This is an open-access article distributed under the terms of the Creative Commons Attribution License. 